PROCEEDINGS OF THE

AMERICAN MATHEMATICAL SOCIETY

Volume 135, Number 3, March 2007, Pages 665-670

S 0002-9939(06)08606-0

Article electronically published on August 28, 2006

\title{
ON THE HARTSHORNE-SPEISER-LYUBEZNIK THEOREM ABOUT ARTINIAN MODULES WITH A FROBENIUS ACTION
}

\author{
RODNEY Y. SHARP
}

(Communicated by Bernd Ulrich)

\begin{abstract}
Let $R$ be a commutative Noetherian local ring of prime characteristic. The purpose of this paper is to provide a short proof of G. Lyubeznik's extension of a result of R. Hartshorne and R. Speiser about a module over the skew polynomial ring $R[x, f]$ (associated to $R$ and the Frobenius homomorphism $f$, in the indeterminate $x$ ) that is both $x$-torsion and Artinian over $R$.
\end{abstract}

\section{INTRODUCTION}

In the theory of tight closure of ideals in a $d$-dimensional commutative (Noetherian) local ring $(R, \mathfrak{m})$ of prime characteristic $p$, the study of properties of the 'top' local cohomology module $H_{\mathfrak{m}}^{d}(R)$ related to the Frobenius homomorphism $f: R \longrightarrow R$ has been a very effective tool (see, for example, K. E. Smith [10, 11]). Some of the properties of $H_{\mathfrak{m}}^{d}(R)$ related to $f$ can be neatly described in terms of a natural structure which $H_{\mathfrak{m}}^{d}(R)$ possesses as a left module over the skew polynomial ring $R[x, f]$; also, it is well known that $H_{\mathfrak{m}}^{d}(R)$ is Artinian as an $R$-module. One can take the view that $H_{\mathfrak{m}}^{d}(R)$ is an important example of a left $R[x, f]$-module that is Artinian as an $R$-module.

In 1977, R. Hartshorne and R. Speiser [2, Proposition 1.11] proved, in the case where the local ring $R$ of characteristic $p$ contains its residue field which is perfect, that, given a left $R[x, f]$-module $H$ that is Artinian as an $R$-module, there exists a non-negative integer $e$ with the following property: whenever $h \in H$ is such that $x^{j} h=0$ for some positive integer $j$, then $x^{e} h=0$.

Twenty years later, G. Lyubeznik [7, Proposition 4.4] proved this result without restriction on the local ring $R$ of characteristic $p$, that is, he was able to drop the hypotheses about the residue field of $R$. Lyubeznik's proof is an application of his substantial theory of $F$-modules.

There is some evidence that the Hartshorne-Speiser-Lyubeznik Theorem can be exploited to good effect in tight closure theory. For example, it has recently been used in 9] to prove that, if $c$ is a test element for a reduced excellent equidimensional local ring $(R, \mathfrak{m})$ of characteristic $p$, then there exists a power of $p$ that is

Received by the editors September 28, 2005.

2000 Mathematics Subject Classification. Primary 13A35, 13E10, 16S36; Secondary 13D45.

Key words and phrases. Commutative Noetherian ring, prime characteristic, Frobenius homomorphism, Artinian module, Frobenius skew polynomial ring.

The author was partially supported by the Engineering and Physical Sciences Research Council of the United Kingdom (grant number EP/C538803/1). 
a test exponent for $c, \mathfrak{a}$ (see [3, Definition 2.2]) for every parameter ideal $\mathfrak{a}$ of $R$ simultaneously.

It therefore seems desirable to have a short proof of the Hartshorne-SpeiserLyubeznik Theorem that does not rely on the theory of $F$-modules. This paper provides one that actually follows the general line of the Hartshorne-Speiser proof.

\section{Left modules OVER THE SKEW POlynomial Ring $R[x, f]$}

1.1. Notation. Throughout the paper, $A$ will denote a general commutative Noetherian ring, and $R$ will denote a commutative Noetherian ring of prime characteristic $p$. In cases where such a ring is assumed to be local, the notation $(A, \mathfrak{m})$ or $(R, \mathfrak{m})$ will indicate that $\mathfrak{m}$ is the maximal ideal.

We shall always denote by $f: R \longrightarrow R$ the Frobenius homomorphism, for which $f(r)=r^{p}$ for all $r \in R$. We use $\mathbb{N}$ and $\mathbb{N}_{0}$ to denote the sets of positive integers and non-negative integers, respectively. We shall work with the skew polynomial ring $R[x, f]$ associated to $R$ and $f$ in the indeterminate $x$ over $R$. Recall that $R[x, f]$ is, as a left $R$-module, freely generated by $\left(x^{i}\right)_{i \in \mathbb{N}_{0}}$, and so consists of all polynomials $\sum_{i=0}^{n} r_{i} x^{i}$, where $n \in \mathbb{N}_{0}$ and $r_{0}, \ldots, r_{n} \in R$; however, its multiplication is subject to the rule

$$
x r=f(r) x=r^{p} x \quad \text { for all } r \in R .
$$

1.2. Definition and remarks. We say that the left $R[x, f]$-module $H$ is $x$-torsionfree if $x h=0$, for $h \in H$, only when $h=0$. The set $\Gamma_{x}(H):=\left\{h \in H: x^{j} h=0\right.$ for some $j \in \mathbb{N}\}$ is an $R[x, f]$-submodule of $H$, called the $x$-torsion submodule of $H$. In general, the $R[x, f]$-module $H / \Gamma_{x}(H)$ is $x$-torsion-free.

\section{The Hartshorne-Speiser Theorem}

As explained in the Introduction, this paper is concerned with the following result of R. Hartshorne and R. Speiser.

2.1. Theorem (Hartshorne-Speiser [2, Proposition 1.11]). Suppose that $R$ is local and contains its residue field which is perfect. Let $H$ be a left $R[x, f]$-module which is Artinian as an $R$-module. Then there exists $e \in \mathbb{N}_{0}$ such that $x^{e} \Gamma_{x}(H)=0$.

G. Lyubeznik [7, Proposition 4.4] proved this result without restriction on the local ring $R$ of characteristic $p$, that is, he was able to drop the hypotheses about the residue field of $R$; his proof is an application of his theory of $F$-modules. The main purpose of this section is to show how one can modify the argument of Hartshorne and Speiser to obtain a short and direct proof of the result in the generality achieved by Lyubeznik. To achieve this aim, we shall establish a generalization of Proposition 1.9 of Hartshorne-Speiser [2].

Our first preparatory result concerns an Artinian module of finite injective dimension over a general local ring $(A, \mathfrak{m})$. Let $E$ denote $E_{A}(A / \mathfrak{m})$, the injective envelope of the simple $A$-module $A / \mathfrak{m}$. Recall that an $A$-module is Artinian if and only if it is isomorphic to a submodule of $E^{t}$, the direct sum of $t$ copies of $E$, for some $t \in \mathbb{N}$. It follows that, if $G$ is an Artinian $A$-module, then, for each $i \in \mathbb{N}_{0}$, the $i$-th term $E_{A}^{i}(G)$ in the minimal injective resolution of $G$ is isomorphic to a direct sum of finitely many copies of $E$. When $J$ is an Artinian injective $A$-module, we shall use the Bass number $\mu^{0}(\mathfrak{m}, J)$ to denote the number of copies of $E$ that occur in a decomposition of $J$ as a direct sum of indecomposable injective $A$-modules. 
2.2. Proposition. Let $G$ be an Artinian module over the local ring $(A, \mathfrak{m})$ such that inj $\operatorname{dim}_{A} G<\infty$.

(i) Let

$$
I^{\bullet}: 0 \longrightarrow I^{0} \stackrel{d^{0}}{\longrightarrow} I^{1} \longrightarrow \cdots \longrightarrow I^{i} \stackrel{d^{i}}{\longrightarrow} I^{i+1} \longrightarrow \cdots
$$

be a finite injective resolution of $G$ in which each term is isomorphic to a direct sum of copies of $E:=E_{A}(A / \mathfrak{m})$. (It should be noted that the minimal injective resolution of $G$ has this property.) Then the integer $\sum_{i=0}^{\infty}(-1)^{i} \mu^{0}\left(\mathfrak{m}, I^{i}\right)$ is independent of the choice of the finite injective resolution $I^{\bullet}$ of $G$ having the stated property. We call this integer the Euler number of $G$, and denote it by $\chi(G)$ (or $\chi_{A}(G)$ when it is desirable to emphasize the local ring $A)$.

(ii) The Euler number $\chi(G)$ of $G$ is non-negative.

(iii) Let $G^{\prime}, \bar{G}$ be further Artinian A-modules of finite injective dimension and suppose that there is an exact sequence $0 \longrightarrow G^{\prime} \longrightarrow G \longrightarrow \bar{G} \longrightarrow 0$ in the category of A-modules and A-homomorphisms. Then

$$
\chi(G)=\chi\left(G^{\prime}\right)+\chi(\bar{G}) .
$$

(iv) When $A$ is complete, the following three conditions are equivalent:

(a) $\left(0::_{A} G\right) \neq 0$;

(b) $\chi(G)=0$;

(c) $\left(0:_{A} G\right)$ contains a non-zerodivisor of $A$.

Proof. (i),(ii),(iii) There is an $A$-homomorphism $\alpha: G \longrightarrow I^{0}$ such that the sequence

$$
0 \longrightarrow G \stackrel{\alpha}{\longrightarrow} I^{0} \stackrel{d^{0}}{\longrightarrow} I^{1} \longrightarrow \cdots \longrightarrow I^{i} \stackrel{d^{i}}{\longrightarrow} I^{i+1} \longrightarrow \cdots
$$

is exact. Note that $E, G, G^{\prime}, \bar{G}$ and all the $I^{j}\left(j \in \mathbb{N}_{0}\right)$ have natural structures as modules over the completion $(\widehat{A}, \widehat{\mathfrak{m}})$ of $A$, and that, when they are given these, there is an $\widehat{A}$-isomorphism $E \cong E_{\widehat{A}}(\widehat{A} / \widehat{\mathfrak{m}})$ and the above-displayed exact sequence provides an injective resolution of $G$ as an $\widehat{A}$-module. Furthermore, $0 \longrightarrow G^{\prime} \longrightarrow G \longrightarrow \bar{G} \longrightarrow 0$ is an exact sequence in the category of $\widehat{A}$-modules and $\widehat{A}$-homomorphisms. It thus follows that it is sufficient to prove parts (i), (ii) and (iii) under the additional assumption that $A$ is complete.

Let $D$ be the functor $\operatorname{Hom}_{A}(\bullet, E)$ on the category of $A$-modules. We use Matlis duality. Since $D(E) \cong A$, application of the functor $D$ to $I^{\bullet}$ yields an exact sequence

$$
\cdots \longrightarrow D\left(I^{i+1}\right) \longrightarrow D\left(I^{i}\right) \longrightarrow \cdots \longrightarrow D\left(I^{0}\right) \longrightarrow D(G) \longrightarrow 0
$$

and this provides a finite free resolution of the finitely generated $A$-module $D(G)$. Moreover, for each $i \in \mathbb{N}_{0}$, the free $A$-module $D\left(I^{i}\right)$ is finitely generated of rank $\mu^{0}\left(\mathfrak{m}, I^{i}\right)$. Thus

$$
\sum_{i=0}^{\infty}(-1)^{i} \mu^{0}\left(\mathfrak{m}, I^{i}\right)=\sum_{i=0}^{\infty}(-1)^{i} \operatorname{rank} D\left(I^{i}\right),
$$

which is just the Euler number $\chi(D(G))$, and so is independent of the choice of finite injective resolution $I^{\bullet}$ of $G$ of the type under consideration (see [8, p. 159], for example). Likewise, the claim in part (ii) now follows from the corresponding statement (see [8, Theorem 19.7], for example) about modules with finite free resolutions, and the claim in part (iii) follows from the well-known fact that $\chi$ is additive on short exact sequences of modules with finite free resolutions. 
(iv) Since the annihilators of $G$ and $D(G)$ are equal, the equivalence of (a), (b) and (c) is now immediate from a theorem of M. Auslander and D. A. Buchsbaum 1]. (see [8, Theorem 19.8], for example).

2.3. Remark. It is a consequence of Proposition 2.2 that, with the notation of that result, $\chi_{A}(G)=\sum_{i=0}^{\infty}(-1)^{i} \mu^{i}(\mathfrak{m}, G)=\sum_{i=0}^{\operatorname{inj} \operatorname{dim} G}(-1)^{i} \mu^{i}(\mathfrak{m}, G)$, because, for each $i \in \mathbb{N}_{0}$, the $i$-th term in the minimal injective resolution of $G$ is isomorphic to the direct sum of $\mu^{i}(\mathfrak{m}, G)$ copies of $E$, and $\mu^{j}(\mathfrak{m}, G)=0$ for all $j>\operatorname{inj} \operatorname{dim}_{A} G$.

We can now establish the promised generalization of Proposition 1.9 of Hartshorne-Speiser [2].

2.4. Proposition (Compare Hartshorne-Speiser [2, Proposition 1.9]). Assume that $(R, \mathfrak{m})$ is a complete regular local ring, and that $H$ is a left $R[x, f]$-module which is Artinian as an $R$-module and such that $R x H=H$. Let $K=\{h \in H: x h=0\}$, an $R[x, f]$-submodule of $H$. Then $\left(0:_{R} K\right) \neq 0$.

Proof. Here, we shall use $R^{\prime}$ to denote $R$ considered as an $R$-module by means of $f$ (at points where care is needed). Also $F$ will denote the Frobenius functor $R^{\prime} \otimes_{R}(\bullet)$ from the category of all $R$-modules and $R$-homomorphisms to the category of all $R^{\prime}$-modules and $R^{\prime}$-homomorphisms.

Since $a x r h=a r^{p} x h$ for $a \in R^{\prime}, r \in R$ and $h \in H$, there is an $R$-homomorphism $\phi: F(H) \longrightarrow H$ for which $\phi(a \otimes h)=a x h$ for all $h \in H$ and $a \in R^{\prime}$. Note that $\phi$ is surjective because $R x H=H$. Note also that, if $h \in K$, then the element $1 \otimes h$ of $F(H)$ lies in $\operatorname{Ker} \phi$. Since $R$ is regular, $f: R \longrightarrow R$ is flat (by E. Kunz [6]), and therefore faithfully flat. The $\mathbb{Z}$-homomorphism $\gamma: K \longrightarrow \operatorname{Ker} \phi$ for which $\gamma(h)=1 \otimes h$ for all $h \in K$ is therefore injective. It is therefore enough for us to show that $\left(0::_{R} \operatorname{Ker} \phi\right) \neq 0$, for if $0 \neq a \in R$ annihilates Ker $\phi$, then, for each $h \in K$, we have $\gamma(a h)=1 \otimes a h=a^{p} \otimes h=a^{p}(1 \otimes h)=0$.

There is a short exact sequence

$$
0 \longrightarrow \operatorname{Ker} \phi \hookrightarrow F(H)=R^{\prime} \otimes_{R} H \stackrel{\phi}{\longrightarrow} H \longrightarrow 0
$$

of $R$-modules and $R$-homomorphisms. By Huneke-Sharp [4, Proposition 1.5], for each injective $R$-module $I$, we have $F(I) \cong I$. Observe that every $R$-module has finite injective dimension because $R$ has finite global dimension. If one applies the exact functor $F$ to the minimal injective resolution for $H$, one can deduce, with the aid of Proposition 2.2, that $F(H)$ is isomorphic to a submodule of the direct sum of finitely many copies of $F(E) \cong E$ and so is Artinian, and that $\chi(F(H))=\chi(H)$. Hence $\operatorname{Ker} \phi$ is an Artinian $R$-module, and it follows from Proposition 2.2(iii) that $\chi(\operatorname{Ker} \phi)=\chi(F(H))-\chi(H)=0$. Hence $\left(0:_{R} \operatorname{Ker} \phi\right) \neq 0$ by Proposition 2.2 (iv).

We shall need the following lemma of Hartshorne and Speiser.

2.5. Lemma (Hartshorne-Speiser [2, Lemma 1.10]). Let $H$ be a left $R[x, f]$-module, and set

$$
K:=\{h \in H: x h=0\},
$$

an $R[x, f]$-submodule of $H$. Suppose that $a \in R$ is such that $a K=0$. Then $a^{2} \Gamma_{x}(H)=0$. 
The short proof, presented in the next theorem, of Lyubeznik's extension of the Hartshorne-Speiser Theorem follows the general line of argument of Hartshorne and Speiser.

2.6. Theorem (G. Lyubeznik [7, Proposition 4.4]; compare Hartshorne-Speiser [2, Proposition 1.11]). Suppose that $(R, \mathfrak{m})$ is local, and let $H$ be a left $R[x, f]$-module which is Artinian as an $R$-module. Then there exists e $\in \mathbb{N}_{0}$ such that $x^{e} \Gamma_{x}(H)=0$.

Proof. Recall the natural $\widehat{R}$-module structure on the Artinian $R$-module $H$ : given $h \in H$, there exists $t \in \mathbb{N}$ such that $\mathfrak{m}^{t} h=0$; for an $\widehat{r} \in \widehat{R}$, choose any $r \in R$ such that $\widehat{r}-r \in \mathfrak{m}^{t} \widehat{R}$; then $\widehat{r} h=r h$. It is easy to see from this that $x \widehat{r} h=\widehat{r}^{p} x h$ for all $h \in H$ and $\widehat{r} \in \widehat{R}$, and we can then use [5, Lemma 1.3] to see that one can assume that $R$ is complete.

Argue by induction on $n:=\operatorname{dim} R$; note that, when $n=0$, the Artinian $R$ module $H$ has finite length and then the claim follows easily. Suppose that $n>$ 0 and assume inductively that the result has been proved when the underlying complete local ring $R$ has dimension smaller than $n$.

Let $k$ be a coefficient field for $R$, and let $r_{1}, \ldots, r_{n}$ be a system of parameters for $R$. Then $R$ is module-finite over the complete regular local ring $k\left[\left[r_{1}, \ldots, r_{n}\right]\right]$, which we denote by $(S, \mathfrak{n})$. Since $\mathfrak{m} \cap S=\mathfrak{n}$, it is clear that each element of $H$ is annihilated by some power of $\mathfrak{n}$. Since $\mathfrak{n} R$ is $\mathfrak{m}$-primary, it contains $\mathfrak{m}^{t}$ for some $t \in \mathbb{N}$, and so $\left(0:_{H} \mathfrak{n}\right)=\left(0:_{H} \mathfrak{n} R\right) \subseteq\left(0:_{H} \mathfrak{m}^{t}\right)$, which is finitely generated over $R$ and therefore over $S$. Thus $H$ is Artinian as an $S$-module.

We can replace $H$ by its $R[x, f]$ - and $S[x, f]$-submodule $\Gamma_{x}(H)$; thus we can assume that $H$ is $x$-torsion. Of course, we can assume that $H \neq 0$.

The descending chain of $S[x, f]$-submodules

$$
H \supseteq S x H \supseteq S x^{2} H \supseteq \cdots \supseteq S x^{i} H \supseteq S x^{i+1} H \supseteq \cdots
$$

of $H$ must eventually stabilize: let $t \in \mathbb{N}_{0}$ be such that $S x^{t} H=S x^{t+j} H$ for all $j \in \mathbb{N}$. Observe that $S x^{t} H=S x\left(S x^{t} H\right)$, and that it is enough to prove the claim for $S x^{t} H$ rather than $H$.

It thus follows that, in order to complete the inductive step, we can (replace $R$ by $S$ and) assume that $R$ is regular (and complete), that $H$ is $x$-torsion and that $H=R x H$.

Let $K=\{h \in H: x h=0\}$. By Proposition 2.4, there exists $0 \neq a \in R$ such that $a K=0$. Therefore $a^{2} \Gamma_{x}(H)=a^{2} H=0$, by Lemma 2.5.

Thus $H$ has a natural structure as a module over the complete local ring $R / R a^{2}$, which has dimension $n-1$. Use $\bar{r}$ to denote the natural image in $R / R a^{2}$ of an element $r \in R$. Then $x \bar{r} h=\bar{r}^{p} x h$ for all $r \in R$ and $h \in H$; it thus follows from [5, Lemma 1.3] that $H$ inherits a structure as left $\left(R / R a^{2}\right)[x, f]$-module, compatible with its $R[x, f]$-module structure; note that $H$ is still Artinian over $R / R a^{2}$ and $x$-torsion, and satisfies $H=\left(R / R a^{2}\right) x H$. Application of the inductive hypothesis therefore completes the proof.

I am grateful to Craig Huneke for pointing out that the argument used in the above proof can be modified to prove the next result.

2.7. Theorem. Suppose that $(R, \mathfrak{m})$ is local, and let $H$ be an $x$-torsion left $R[x, f]$ module which is Artinian as an $R$-module. If $H=R x H$, then $H$ has finite length as an $R$-module. 
Proof. Only a sketch is presented here, as the strategy used is very similar to that used in the above proof of Theorem 2.6.

One can assume that $R$ is complete; then argue by induction on $n:=\operatorname{dim} R$, the result being easy when $n=0$. For the inductive step, in the situation where $n>0$, again let $k$ be a coefficient field for $R$, let $r_{1}, \ldots, r_{n}$ be a system of parameters for $R$, and let $S$ be the complete regular local ring $k\left[\left[r_{1}, \ldots, r_{n}\right]\right]$. We can again use the fact that $R$ is module-finite over $S$ to see that $H$ is Artinian as an $S$-module, so that there exists $t \in \mathbb{N}_{0}$ such that $S x^{t} H=S x^{t+j} H$ for all $j \in \mathbb{N}$.

Thus the left $S[x, f]$-module $S x^{t} H$ is $x$-torsion, Artinian as an $S$-module, and such that $S x^{t} H=S x\left(S x^{t} H\right)$. Let $e_{1}=1, e_{2}, \ldots, e_{h}$ generate $R$ as an $S$-module. If we could prove that $S x^{t} H$ is of finite length as an $S$-module, then, since $H=R x H$, it would follow that

$$
H=R x^{t} H=\sum_{i=1}^{h} S e_{i} x^{t} H=\sum_{i=1}^{h} e_{i} S x^{t} H
$$

is finitely generated as an $S$-module, and therefore as an $R$-module. It follows that, in order to complete the inductive step, we can (replace $R$ by $S$ and) assume that $R$ is regular (and complete). The proof can now be completed by an argument almost identical to that in the last two paragraphs of the above proof of Theorem 2.6.

2.8. Remark. Note that an extension of the Hartshorne-Speiser-Lyubeznik Theorem to non-local situations is proved in [9, Corollary 1.8]. There it is proved that, if $R$ is merely a commutative Noetherian ring (of characteristic $p$ ), and $H$ is a left $R[x, f]$-module which is Artinian as an $R$-module, then there exists $e \in \mathbb{N}_{0}$ such that $x^{e} \Gamma_{x}(H)=0$.

\section{REFERENCES}

1. M. Auslander and D. A. Buchsbaum, Codimension and multiplicity, Annals of Math. 68 (1958) 625-657. MR0099978 (20:6414)

2. R. Hartshorne and R. Speiser, Local cohomological dimension in characteristic p, Annals of Math. 105 (1977) 45-79. MR0441962 (56:353)

3. M. Hochster and C. Huneke, Localization and test exponents for tight closure, Michigan Math. J. 48 (2000) 305-329. MR.1786493 (2002a:13001)

4. C. Huneke and R. Y. Sharp, Bass numbers of local cohomology modules, Transactions Amer. Math. Soc. 339 (1993) 765-779. MR1124167 (93m:13008)

5. M. Katzman and R. Y. Sharp, Uniform behaviour of the Frobenius closures of ideals generated by regular sequences, J. Algebra, 295 (2006) 231-246. MR2188859

6. E. Kunz, Characterizations of regular local rings of characteristic p, Amer. J. Math. 91 (1969) 772-784. MR0252389 (40:5609)

7. G. Lyubeznik, F-modules: applications to local cohomology and D-modules in characteristic $p>0$, J. reine angew. Math. 491 (1997) 65-130. MR.1476089 (99c:13005)

8. H. Matsumura, Commutative ring theory, Cambridge Studies in Advanced Mathematics 8, Cambridge University Press, 1986. MR0879273 (88h:13001)

9. R. Y. Sharp, Tight closure test exponents for certain parameter ideals, Michigan Math. J., to appear (arXiv math.AC/0508214).

10. K. E. Smith, Tight closure of parameter ideals, Inventiones mathematicae 115 (1994) 41-60. MR.1248078 (94k:13006)

11. K. E. Smith, Test ideals in local rings, Transactions Amer. Math. Soc. 347 (1995) 3453-3472. MR:1311917 (96c:13008)

Department of Pure Mathematics, University of Sheffield, Hicks Building, Sheffield S3 7RH, United Kingdom

E-mail address: R.Y.Sharp@sheffield.ac.uk 